\title{
Temporal Aquaporin 11 Expression and Localization during Preimplantation Embryo Development
}

\author{
Jae-Won Park and ${ }^{\dagger}$ Yong-Pil Cheon \\ Division of Developmental Biology and Physiology, School of Biosciences and Chemistry, Sungshin University, \\ Seoul 147-742, Korea
}

\begin{abstract}
Environmental conditions during early mammalian embryo development are critical and some adaptational phenomena are observed. However, the mechanisms underlying them remain largely masked. Previously, we reported that AQP5 expression is modified by the environmental condition without losing the developmental potency. In this study, AQP11 was examined instead. To compare expression pattern between in vivo and in vitro, we conducted quantitative RT-PCR and analyzed localization of the AQP11 by whole mount immunofluorescence. When the fertilized embryos were developed in the maternal tracts, the level of Aqp11 transcripts was decreased dramatically until 2-cell stage. Its level increased after 2-cell stage and peaked at 4-cell stage, but decreased again dramatically until morula stage. Its transcript level increased again at blastocyst stage. In contrast, the levels of Aqp11 transcript in embryos cultured in vitro were as follows. The patterns of expression were similar but the overall levels were low compared with those of embryos grown in the maternal tracts. AQP11 proteins were localized in submembrane cytoplasm of embryos collected from maternal reproductive tracts. The immunereactive signals were detected in both trophectoderm and inner cell mass. However, its localization was altered in in vitro culture condition. It was localized mainly in the plasma membrane of the blastocysts contacting with external environment. The present study suggests that early stage embryo can develop successfully by themselves adapting to their environmental condition through modulation of the expression level and localization of specific genes like AQP11.
\end{abstract}

Key words: Aquaporin 11, Early stage embryo, Developmental potency, Adaptation

\section{INTRODUCTION}

An early stage adaptational development has been suggested and evaluated in some invertebrate animals. In oyster Crassostrae gigas, temperature influences histone methylation and mRNA expression of the Jmj-C histone-demethylase orthologues (Fellous et al., 2015). In mammalian embryo, such adaptational development is not well determined. Recently, using mouse embryo culture system, Bell et al
(2009) showed successful development in the stressful hyperosmotic stress and changing of profiles in expressed genes. Additional evidences are reported in mouse embryos (Park et al., 2014). Such plastic responses during embryo development are thought as a strategy to cope with immediate or predicted circumstances, to maximize fitness in the context of the range of environments potentially faced (Gluckman et al., 2011).

One of the survival factors is a well maintained home-

\footnotetext{
Manuscript received 5 March 2015, Received in revised form 16 March 2015, Accepted 23 March 2015

$\dagger$ Corresponding Author : Yong-Pil Cheon, Division of Developmental Biology and Physiology, School of Biosciences and Chemistry, Sungshin University, 173-1 Dongseondong 3ga, Seongbuk-gu, Seoul 136-742, Korea. Tel. : +82-2-920-7639, 2766, Fax : +82-2-920-2047, E-mail : ypcheon@ sungshin.ac.kr

This is an Open Access article distributed under the terms of the Creative Commons Attribution Non-Commercial License (http:// creativecommons.org/licenses/by-nc/3.0) which permits unrestricted non-commercial use, distribution, and reproduction in any medium, provided the original work is properly cited.
} 
ostasis in cytoplasm. Cells have recognition systems to extra- and intracellular environment. Small changes in cell volume and pressure serve as important signals for cellular responses such as prolife-ration, death and migration. Homeostasis in cytoplasm is maintained through exchanges with external environment. Therefore, cell must adapt to osmotic shocks and actively adjust the adaptation level (Jiang and Sun, 2013). Homeostatic internal environment of cytoplasm and blastomere is depending on the amount of water, ion, nutrients, and organic molecules. Mechanosensitive channels, active ion pumps, and water channels (aquaporins, AQPs) are a key to understanding the overall mechanical response for cellularization during early stage embryo development.

Thirteen AQPs (AQP0-12) are revealed and distributed in different organs (Hua et al., 2013). AQPs can be distinguished their sequence homology and permeable functions as following (Gonen and Walz, 2006): AQP0, -1, -2, -4, -5, -6 , and -8 are aquaporins and highly selective for water molecules, although AQP6 and AQP8 only belong to this group based on their sequence, since AQP6 is an anion channel (Yasui et al., 1999) and AQP8 is permeable to urea (Ishibashi et al., 1997). AQP 3, 7, 9, and 10 are aquaglyceroporins allowing passage of small solute (for example, glycerol, urea, purines, pyrimidines, carbamides, and polyols) with AQP9 exhibiting the broadest substrate specificity (Borgnia et al, 1999; Ishibashi et al, 2002; Gonen and Walz, 2006; Itoh et al., 2005). The nucleotide homology of AQP11 and -12 is not much high compared with the other AQPs (Calvanese et al., 2013). The substrate specificities for the superaquaporins AQP11 and AQP12 have not yet fully determined (Ishibashi et al, 2002; 2014).

A few studies were performed to detect Aqp expression in early stage embryos. AQP5 is known to maintain proper osmolality of the secreted saliva and lens (Ma et al., 1999; Kumari and Varadaraj, 2013) and is suggested to involve in embryo development. AQP5 may control the water transport with other AQPs during early developmental stages because AQP5 is highly permeable to water but not to small organic and inorganic molecules (Huang et al., 2006; Park et al., 2014). During blastocoel formation, AQP3 expression is under the control of p38 MAPK and it is critical in this process (Bell and Watson, 2013).

It has been suggested that AQP11 could work as a sort of intracellular aquaporin in certain tissues and have a role in intravesicular homeostasis of kidney proximal tubule cells, mammalian spermatogenesis and kidney, and salivary gland development (Magni et al., 2006). AQP11's expressed in developing mouse submandibular gland (Larsen et al., 2010) and human early stage embryos (Xiong et al., 2013). AQP11 is not fully characterized but detected in blastocyst in mouse (Offenberg and Thomsen, 2015). Previously in our laboratory screened the expression of the AQPs family during early stage embryos. However, whether the expression is altered by environment is not evaluated in early stage embryos, so far. In this study, we evaluate the expression profiles during early stage embryo and modification of the levels of AQP11 transcripts and proteins.

\section{MATERIALS AND METHODS}

\section{Experimental animals}

Experiment animals were maintained and treated according to the Guide for the Care and Use of Laboratory animals published by National Institutes of Health and under the Experimental Animals Committee of Sungshin University. Animals were maintained under standard conditions at animal house in Sungshin university with diurnal rhythm kept under the 14L : 10D schedule with light-on at 06:00 and clean room system. Animals were fed a standard rodent diet and water ad libitum from weaning at 21 days after birth.

\section{Superovulation induction and embryo collection}


Six to eight week-old female CD-1 mice were used in this study. Animals were injected with 5 IU/0.1 mL PMSG (Sigma) followed by $5 \mathrm{IU} / 0.1 \mathrm{~mL}$ CG (Sigma) $48 \mathrm{hr}$ later, and mated with CD-1 males. Next morning, the day of vaginal plug was designated Day 1 of pregnancy. Time posthCG was standard to measure the developmental stage embryo which were collected at $18 \mathrm{hr}$ unfertilized egg (UF); 21-24 hr pronucleus stage embryo (PN); $48 \mathrm{hr}$, 2-cell (2-C); 60 hr, 4-cell (4-C); 65-68 hr, 8-cell (8-C); 80-85 hr, morula (Mor); 96-100 hr, blastocyst (Bla). To collect in vivo embryos, we flushed the oviduct and uterus using flushing medium 0.4\% BSA BWW. Flushed 2-cell stage embryo were cultured to blastocyst. Collected embryos were quickfrozen in a small amount of medium using liquid nitrogen. They were stored at $-80^{\circ} \mathrm{C}$ until RNA extraction.

\section{Embryo culture}

The collected healthy two cell embryos were cultured in the $10 \mu \mathrm{L}$ drops of BWW medium contacting $0.4 \%$ bovine serum albumin (BSA). The embryo were incubated at $37^{\circ} \mathrm{C}$ with $5 \% \mathrm{CO}_{2}$ in air until $120 \mathrm{hr}$ post hCG injection. The embryo development was evaluated every 12 or $24 \mathrm{hr}$ under the inverted microscope (Olympus, $1 \times 70$ ).

\section{Total RNA extraction and first strand cDNA synthesis}

Total RNA of embryos was extracted by using RNeasy ${ }^{\circledR}$ Micro Kit (QIAGEN, CA USA) according to the manual of manufacturer. Total RNA $5 \mu \mathrm{g}$ were used to perform reverse transcription. First strand cDNA synthesis was conducted by AffinityScript cDNA Synthesis Kit (Agilent,
CA, USA) according to the manufacturer's instructions. Briefly, reaction reagents are total RNA $5 \mu \mathrm{g}, 5.0 \mu \mathrm{L}$ Accuscript buffer (10×), $6.0 \mu \mathrm{L}$ oligo dT primer $(0.5 \mu \mathrm{g} / \mu \mathrm{L}), 1.0 \mu \mathrm{L}$ random primer $(0.1 \mu \mathrm{g} / \mu \mathrm{L}), 2 \mu \mathrm{L}$ dNTP mix $(100 \mathrm{mM}), 1$ $\mu \mathrm{L}$ RNase-free water. Reaction mixture was incubated at $65^{\circ} \mathrm{C}$ for $5 \mathrm{~min}$, placed the tube at $\mathrm{RT}$ to allow the primers to anneal to RNA for 10 min and then, added $4.0 \mu \mathrm{L}$ DTT (100 mM), $1 \mu \mathrm{L}$ Accuscript multiple temperature RT, $2 \mu \mathrm{L}$ RNase block ribonuclease inhibitor $(40 \mathrm{U} / \mathrm{mL})$. The mixture was incubated at $42^{\circ} \mathrm{C}$ for $1 \mathrm{hr}$ to synthesis and then $72^{\circ} \mathrm{C}$ for 15 min to terminate cDNA synthesis.

\section{Real Time RT-PCR}

Transcripts of target gene were amplified using real time PCR (Takara, TP800) and the specific primer (Table 1). For quantitative RT-PCR (qPCR) was performed using SYBR Premix Ex Taq ${ }^{\mathrm{TM}}$ (TakaRa, Japan) and Thermal Cycler Dice Real Time System TP800 (TaKaRa, Japan) as described previously (Park et al., 2014). Each reaction was run in triplicate and consisted of $1.0 \mu \mathrm{L}$ cDNA. Dissociation curves were run on all reactions to ensure amplification of a single product with the appropriate melting temperature. The thermal cycling conditions were : $95^{\circ} \mathrm{C}$ for $1 \mathrm{~min}, 59^{\circ} \mathrm{C}$ for $30 \mathrm{~s}, 72^{\circ} \mathrm{C}$ for $1 \mathrm{~min}$, and for 45 cycles. Data were analyzed by the $\Delta \Delta$ comparative threshold cycle (CT) method.

\section{Whole mount immunofluorochemistry of the mouse} embryo

Whole mount immunofluorochemistry was followed

Table 1. Sequences of sense (S) and antisense (AS) primers (5'-3') for real-time RT-PCR

\begin{tabular}{ccl}
\hline \hline Genes & Gene Bank Access No. & \multicolumn{1}{c}{ Sequneces } \\
\hline \multirow{2}{*}{ Aqp11 } & NM_009701.4 & $\begin{array}{l}\text { S : CTC ATG CTG CTG TTC GTG CTG TTC } \\
\text { AS : GCC ATG CAC CAA GGA AAA GAA }\end{array}$ \\
\hline \multirow{2}{*}{ Ppia } & NM_008907.1 & $\begin{array}{l}\text { S : CCACCGTGTTCTTCGACATCA } \\
\text { AS : GATGCCAGGACCTGTATGCTTTAG }\end{array}$ \\
\hline
\end{tabular}


previous report (Park et al., 2014). The in vivo collected and in vitro cultured embryos were fixed in $4 \%$ paraformaldehyde in PBS containing 3.7\% picric acid in phosphatebuffered saline (PBS) for $30 \mathrm{~min}$. Permeabilzation was conducted by $0.2 \%$ PBST (PBS containing $0.2 \%$ Triton $\mathrm{X}$ 100) for $3 \mathrm{hr}$. The embryos were blocked in $0.1 \%$ PBST containing $10 \%$ normal serum for $1 \mathrm{hr}$ at RT. After then, the embryos were incubated with rabbit polyclonal antiAQP5 (Santa Cruz Biotechnology, Santa Cruz, CA, USA) at a 1:200 dilution at $4{ }^{\circ} \mathrm{C}$ overnight. Embryos were incubated with fluorescence 2nd antibody $\mathrm{Cy}^{\mathrm{TM}} 3$ conjugated AffiniPure (Jackson ImmunoResearch, USA) diluted 1:200 in PBS containing $2 \%$ BSA for $2 \mathrm{hr}$ at RT. To stain the nuclear of embryos, Hochest33238 was used. Using dot slide, embryos were prepared for confocal imaging analysis. For negative controls, we deleted primary antibodies. Finally, fluorescent images were analyzed by Zeiss LSM 700 laser scanning microscopes with ZEN software

\section{Statistics}

Quantitative RT-PCR was conducted at least in triplicate. Differences in the mean values of AQP cDNA copies in the in vivo and in vitro embryos were analyzed using an unpaired $t$-test and ANOVA. Results were presented as Mean \pm SD. Immunofluorescence staining was performed to each group that included in 7 embryos.

\section{RESULTS}

\section{Expression patterns Aqp11 transcripts in vivo}

Firstly, the level of Aqp11 transcript was analyzed in ovulated oocyte and developing embryos in reproductive tracts using real-time PCR technology. The level of Aqp11 transcript was very high in ovulated oocytes compared with the other cleaving embryos. Maternal Aqp11 transcript decreased dramatically until 2-cell stage (Fig. 1). Its level was sharply increased after 2-cell and peaked at 4-cell

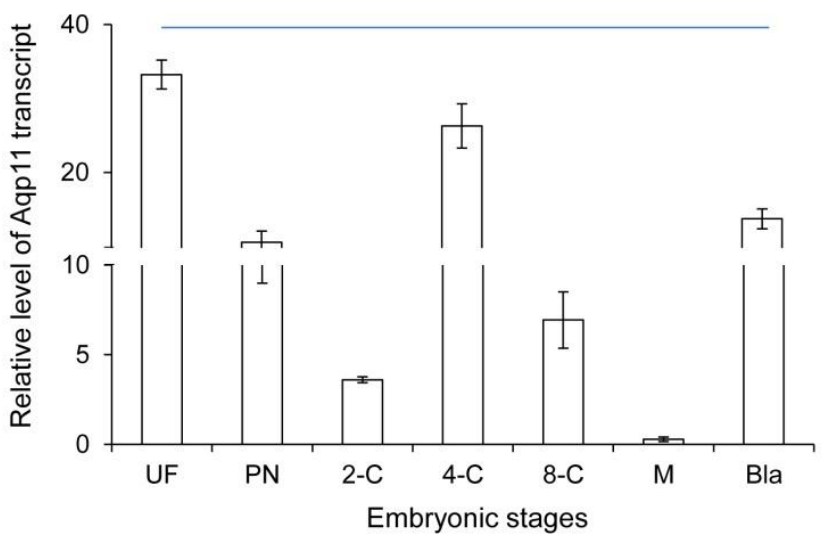

Fig. 1. Relative Aqp11 mRNA levels in preimplantation stage embryos in vivo. Real-time RT-PCR was used to determine the relative mRNA levels of Aqp11 in unfertilized oocyte (UF), pronucleus stage fertilized oocyte (PN), 2-cell (2-C), 4-cell (4-C), 8cell (8-C), morula (Mor), and blastocyst (Bla) which were developed in vivo. Ten embryos were used for real-time RT-PCR per embryonic stage and repeated 3 times in all stages. * Significant difference among stages $(p<0.05$, ANOVA).

stage. After that stage zygotic Aqp11 transcript was decreased until morula stage. The expression levels were again sharply increased at blastocyst stage (Fig. 1).

These results showed that maternal Aqp11 is used at oocyte and zygotic Aqp11 is expressed and peaked at 4cell stage and blastocyst stages.

\section{Differential expression patterns and levels of} Aqp 11 transcripts in vitro

We analyzed the expression levels of zygotic Aqp11 during in vitro culture. 2-cell stage embryos were cultured under the BWW and standard culture condition. Aqp11 transcripts were detected in all the examined stage compared with the same stage in vivo embryos. However, the profile patterns were similar with them of in vivo embryos (Fig. 2). These results indicate that the expression patterns were not changed in Aqp11 expression but the levels of expression or degradation were modified by in vitro culture. 


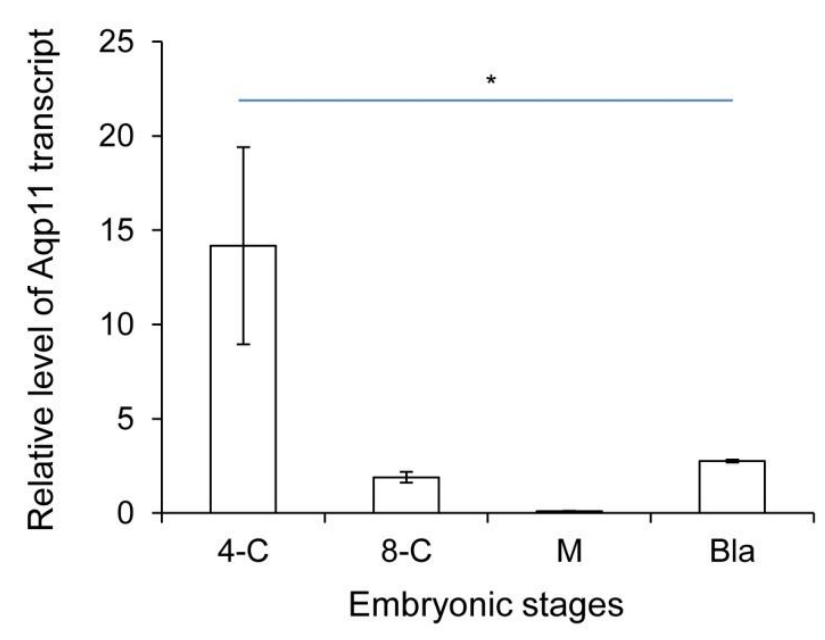

Fig. 2. Relative Aqp11 mRNA levels in preimplantation stage embryos which were cultured in vitro. Real-time RT-PCR was used to determine the relative mRNA levels of Aqp11 in 4-cell (4-C), 8cell (8-C), morula (Mor), and blastocyst (Bla) which were cultured in vitro from 2-cell stage. Ten embryos were used for real-time RT-PCR per embryonic stage and repeated 3 times in all stages. * Significant difference among stages $(p<0.05$, ANOVA).

3. Localization of AQP11 in in vivo early stage embryos and modification by in vitro culture

To evaluate the possible role AQP11 in early embryo, we localized AQP11 with whole mount immunofluorescence method. AQP11 mainly localized in submembranous cytoplasmic areas of blastomeres. It also localized in both trophectoderm and inner cell. By the in vitro culture, its localization was dramatically changed. Until morula stage, AQP11 localized both in apical membrane and cortex of blastomere but the immune-reactive signal was much strong in apical membrane. During blastocyst stage, it was evenly localized in cytoplasm.

\section{DISCUSSION}

Previously mRNAs encoding AQP0 - 12 were analyzed in preimplantation mouse or human embryo (Offenberg et

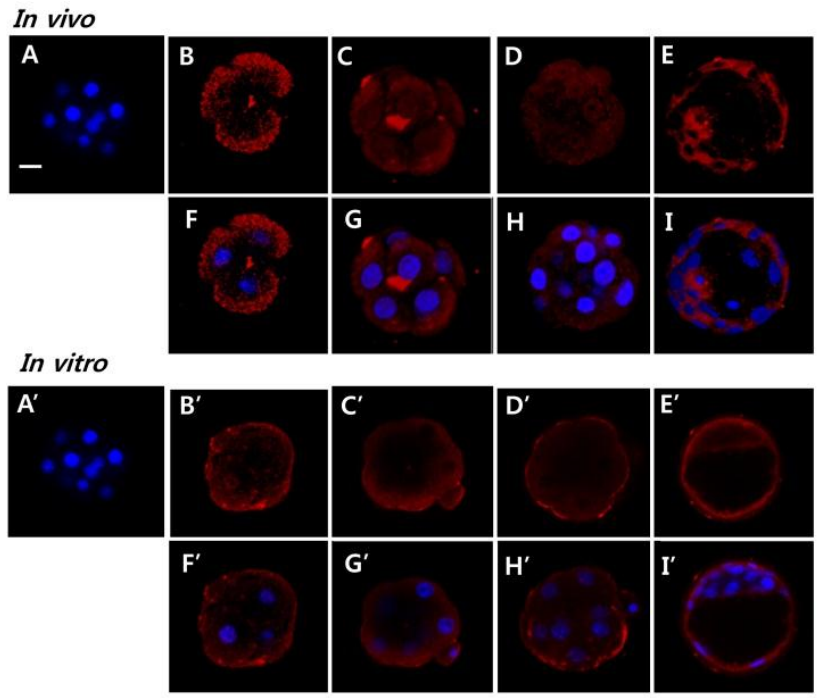

Fig. 3. Laser scanning confocal microscopy image of AQP11 in vivo (A-I) and in vitro (A'-I') developed embryos. The localization of AQP11 in embryo was examined by whole mount immunofluorescence. A and A': negative control; B and B': 4-cell stage embryo; C and C': 8-cell stage embryo; D and D': morula stage embryo; E and E': blastocyst stage embryo; A-D and A'-D': red (anti-AQP11); F-I and F'-I': merged image (blue: Hochest33238); A-I: in vivo embryos; A'-I': in vitro cultured embryos from 2-cell stage; scale bar $=20 \mu \mathrm{m}$. No primary antibody negative control (A and $\mathrm{A}^{\prime}$ ).

al., 2000; Nong et al., 2013; Xiong et al., 2013). Based on them, it has been suggested that multiple AQPs are expressed in their early stage human embryos (Offenberg et al., 2000; Xiong et al., 2013) and that AQP3 and AQP7 may play a role in preimplantation mouse embryo development (Nong et al., 2013; Xiong et al., 2013). So far, Aqp11 expression is not well evaluated in preimplantation stage embryo. This study evaluated the expression of Aqp11 and localization of its products for the first time in all preimplanation stages. In addition, Aqp11 transcripts were detected in ovulated oocytes. This maternal Aqp11 sharply decreased until 2-cell stage and zygotic Aqp11 expressed after 2-cell stage. During early stage, the peak of Aap11 transcript level was observed at 4-cell stage and blastocyst stage. It 
indication thatAQP11 may have a role in early embryo development.

Blastocoel formation is driven by the expression of specific sets of gene products: the E-cadherin-catenin cell adhesion family, the tight junction gene family, the $\mathrm{Na} / \mathrm{K}$ ATPase gene family and Aqp gene family (Andrew and Barcroft, 2001; Watson and Barcroft, 2001). Although the role of AQP11 in blastocoel formation has not been evaluated, its mRNA and protein levels suggest some role. Interestingly, AQP11 was mainly localized in cytoplasm but not in apical membrane in both embryos developed in maternal tracts and cultured in vitro. Besides, AQP11 was mainly localized in submembrane cytoplasm in blastomeres of 4cell, 8-cell, and morula developing within the maternal tracts. Immunohistological studies have shown that AQP11 may work as a sort of intracellular AQP molecule in certain tissues, such as kidney and brain (Morishita et al., 2005; Ikeda et al., 2011). AQP11 also plays significant role in mammalian spermatogenesis and salivary gland development (Larsen et al., 2010; Yeung and Cooper, 2010). Thus AQP11 is thought to be an important intracellular molecule for the development of certain organs. Together, our results showed that AQP11 does not work directly in water transport through apical membrane during early development but work as an important intracellular molecule for early stage embryo development.

Cells containing mutant AQP11 have significantly higher osmotic water permeability (Takahashi et al., 2014). AQP11 expressed in Xenopus oocytes had no water permeability at all (Gorelick et al., 2006), whereas stopped-flow light scattering experiments using reconstituted AQP11 on proteoliposomes revealed normal water permeability (Yakata et al., 2007). Nevertheless, a recent study showed that vesicles incorporating AQP11 directly formed from Sf9 cell membranes had appreciable water permeability (Yakata et al., 2011). AQP11 shows slow water conduction. The water permeability of AQP11 is 8-fold lower than that of AQP1 (Yakata et al.,
2011). It also works as glycerol channel in adipocytes (Madeira et al., 2014). Interestingly, this study revealed that the localization of AQP11 was changed by in vitro culture when compared with that of in vivo embryo. The localization was shifted from cytoplasm to apical membrane and submembrane area in blastomeres of 4-cell, 8-cell, and morula. However, it was not detected at blastocyst stage. Based on them, the localization of AQP11 in the apical membrane suggests that in vitro cultured early stage embryos direct the localization of AQP11 for the environmental adaptation without losing the developmental potency.

In summary, the maternal Aqp11 transcript dramatically decreased until 2-cell stage, and zygotic transcript showed fluctuation, and peaked at both 4-cell stage and blastocyst stages. Its translation product AQP11 mainly located in cytoplasm in all stages of embryos in maternal tracts. However, its localization was modified by in vitro culture, and it mainly localized in apical membrane and submembrane area of blastomere at 4-cell, 8-cell, and morula. It located mainly in cytoplasm at blastocyst stages. Put together, our results suggest that expression of Aqp11 can be modulated by environmental changes and early stage embryos themselves adapt to their microenvironment to achieve developmental competence.

\section{ACKNOWLEDGEMENTS}

This works was supported by Basic Science Research Program through the National Research Foundation of Korea (NRF) funded by the Ministry of Education, Science and Technology (NRF-2012R1A1A2003084).

\section{REFERENCES}

Andrew J, Barcroft LC (2001) Regulation of blastocyst formation. Fron Bio:d708-730.

Bell CE, Lariviere NMK, Watson PH, Watson AJ (2009) 
Mitogen-activated protein kinase (MAPK) pathways mediate embryonic responses to culture medium osmolarity by regulating aquaporin 3 and 9 expression and localization, as well as embyonic apoptosis. Hum Reprod 24:1373-1386.

Borgnia M, Nielsen S, Engel A, Agre P (1999) Cellular and molecular biology of the aquaporin water channels. Annual Rev Biochem 68:425-458.

Calvanese L, Pellegrini-Calace M, Oliva R (2013) In silico study of human aquaporin AQP11 and AQP12 channels. Protein Sci 22:455-466.

Fellous A, Favrel P, Riviere G (2015) Temperature influences histone methylation and mRNA expression of the JmjC histone-demethylase orghologues during the early development of the oyster Crassostrea gigas. Mar Genomics 19:23-30.

Gluckman PD, Hanson MA, Low FM (2011) The role of developmental plasticity and epigenetics in human health. Birth Defects Res C Embryo Today 93:12-18.

Gonen T, Waltz T (2006) The structure of aquaporins. Quarterly Rev Biophys 39:361-396.

Gorelick DA, Praetorius J, Tsunenari T, Nielsen S, Agre P (2006) Aquaporin-11: a channel protein lacking apparent transport function expressed in brain. BMC Biochem 7:14

Hua Y, Jiang W, Zhang W, Shen Q, Chen M, Zhu X (2013) Expression and significance of aquaporins during pregnancy. Front Biosci 18:1373-1383.

Huang HF, He RH, Sun CC, Zhang Y, Meng QX, Ma YY (2006) Funciton of aquaporins in female and male reproductive systems. Hum Reprod Updata 12:785-795.

Ikeda M, Andoo A, Shimono M, Takamatsu N, Taki A, Muta K, Matsushita W, Uechi T, Matsuzaki T, Kenmocki N, Takata K, Sasaki S, Ito K, Ishibashi K (2011) The NPC motif of aquaporin-11, unkike the NPA motif of known aquaporins, is essentai lfor full expression of molecular function. J Biol Chem 286:3342-3350.

Ishibashi K, Kuwahara M, Gu Y, Kageyama Y, Tohsaka A,
Suzuki F, Marumo F, Sasaki S (1997) Cloning and funcitonal expression of a new water channel abundantly expressed in the testis permeable to water, glycerol, and urea. J Biol Chem 272:20782-20789.

Ishibashi K, Morinaga T, Kuwahara M, Sasaki S, Imai M (2002) Cloning and identificaiton of a new member of water channel (AQP10) as an aquaglyceroporin. Biochemica et Biophysica Acta 1576:335-340.

Ishibashi K, Tanaka Y, Morishita Y (2014) The role of mammalian superaquaporins inside the cell. Biochim Biophys Acta 1840:1507-1512.

Itoh T, Rai T, Kuwahara M, Ko SB, Uchida S, Sasaki S, Ishibashi K (2005) Identification of a novel aquaporin, AQP12, expressed in pancreatic acinar cells. Biochem Biophys Res Commun 330:832-838.

Kumari SS, Varadaraj K (2013) Aquaporin 5 knockout mouse lens develops hyperglycemic cataract. Biochem Biophys Res Com 441:333-338.

Larsen HS, Ruus AK, Schreurs O, Galtung HK (2010) Aquaporin 11 in the developing mouse submanibular gland. Eur J Oral Sci 118:9-13.

Ma T, Song Y, Gillespie A, Carlson EJ, Epstein CJ, Verkman AS (1999) Defective secretion of saliva in transgenic mice lacking aquaporin-5 water channels. J Biol Chem 274:20071-20074.

Madeira A, Fernandez-Veledo S, Camps M, Zorzano A, Moura TF, Ceperuelo-Mallafre V, Vendrell J, Soveral G (2014) Human aquaporin-11 is a water and glycerol channel and localized in the vicinity of lipid droplets in human adipocytes. Obesity 22:2010-2017.

Magni F, Sarto C, Ticozzi D, Soldi M, Bosso N, Mocarelli P, Kienle MG (2006) Proteomic knowledge of human aquaporins. Proteomics 6:5637-5649.

Morishita Y, Matsuzaki T, Hara-chikuma M, Andoo A, Shimono M, Matsuki A, Kobayashi K, Ikeda M, Yamamoto T, Verkman A, Kusano E, Ookawara S, Takata K, Sasaki S, Ishibashi K (2005) Disruption of 
aquaporin-11 produces polycystic kidneys following vacuolization of the proximal tubule. Mol Cell Biol 25:7770-7779.

Nong YQ, Liu FH, Chen Y, Wang F. 2013. The expression and distribution of aquaprin 3 in mouse embryos before and after vitrification. J Assist Reprod genet 30:601-606

Offenberg H, Barcroft LC, Caveney A, Viuff D, Thomsen PD, Watson AJ (2000) mRNAs encoding aquaporins are present during murine preimplantation development. Mol Reprod Dev 57:323-330.

Offenberg H, Thomsen PD (2015) Functional challenge affects aquaporin mRNA abundance in mouse blastocysts. Mol Reprod Dev 71:422-430.

Park JW, Shin YK, Cheon YP (2014) Adaptive transition of aquaporin 5 expression and localization during preimplantation embryo developmenty in vitro culture. Dev Reprod 18:153-160.

Takahashi S, Muta K, Sonoda H, Kato A, Abdeen A, Ikeda M (2014) The role of cysteine 227 in subcellular localization, water permeability, and multimerization of aquaporin11. FEBS Open Bio 4:315-320.
Watson AJ, Barcroft LC (2001) Regulation of blastocyst formation. Front Biosci 6:D708-D730.

Xiong Y, Tan YJ, Xiong YM, Huang YT, Hu XL, Lu YC, Ye YH, Wang TT, Zhang D, Jin F, Huang HF, Sheng JZ (2013) Expression of aquaporins in human embryos and potential role of $\mathrm{AQP} 3$ and $\mathrm{AQP7}$ in preimplantation mouse embryo development. Cell Physiol Biochem 31: 649-658.

Yakata K, Hiroaki Y, Ishibashi K, Sohara E, Sasaki S, Mitsuoka K, Fujiyoshi Y (2007) Aquaporin-11 containing a divergent NPA motif has normal water channel activity. Biochim Biophys Acta 1768:688-693.

Yakata K, Tani K, Fujiyoshi Y (2011) Water permeability and characterization of aquaporin-11. J Struct Biol 174 $315-320$

Yasui M, Hazama A, Kwon TH, Nielsen S, Guggino WB, Agre P (1999) Rapid gating and anion permeability of an intracellular aquaporin. Nature 402:184-187.

Yeung CH, Cooper TG (2010) Aquaporin AQP11 in the testis: molecular identity and association with the processing of residual cytoplsm of elongated spermatids. Reproduction 139:209-216. 